http://revistas.ucr.ac.cr/index.php/ingenieria

www.ucr.ac.cr / ISSN 1409-2441
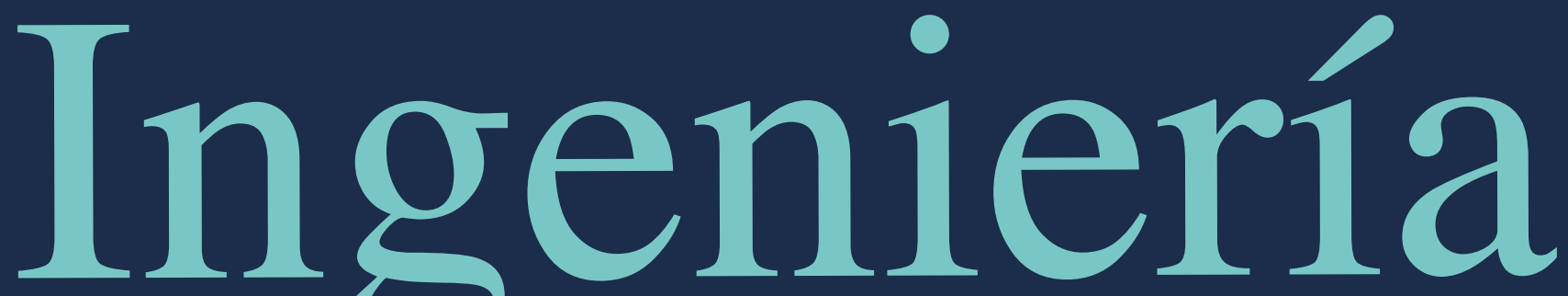

Revista de la Universidad de Costa Rica JULIO / DICIEMBRE 2019 - VOLUMEN 29 (2)

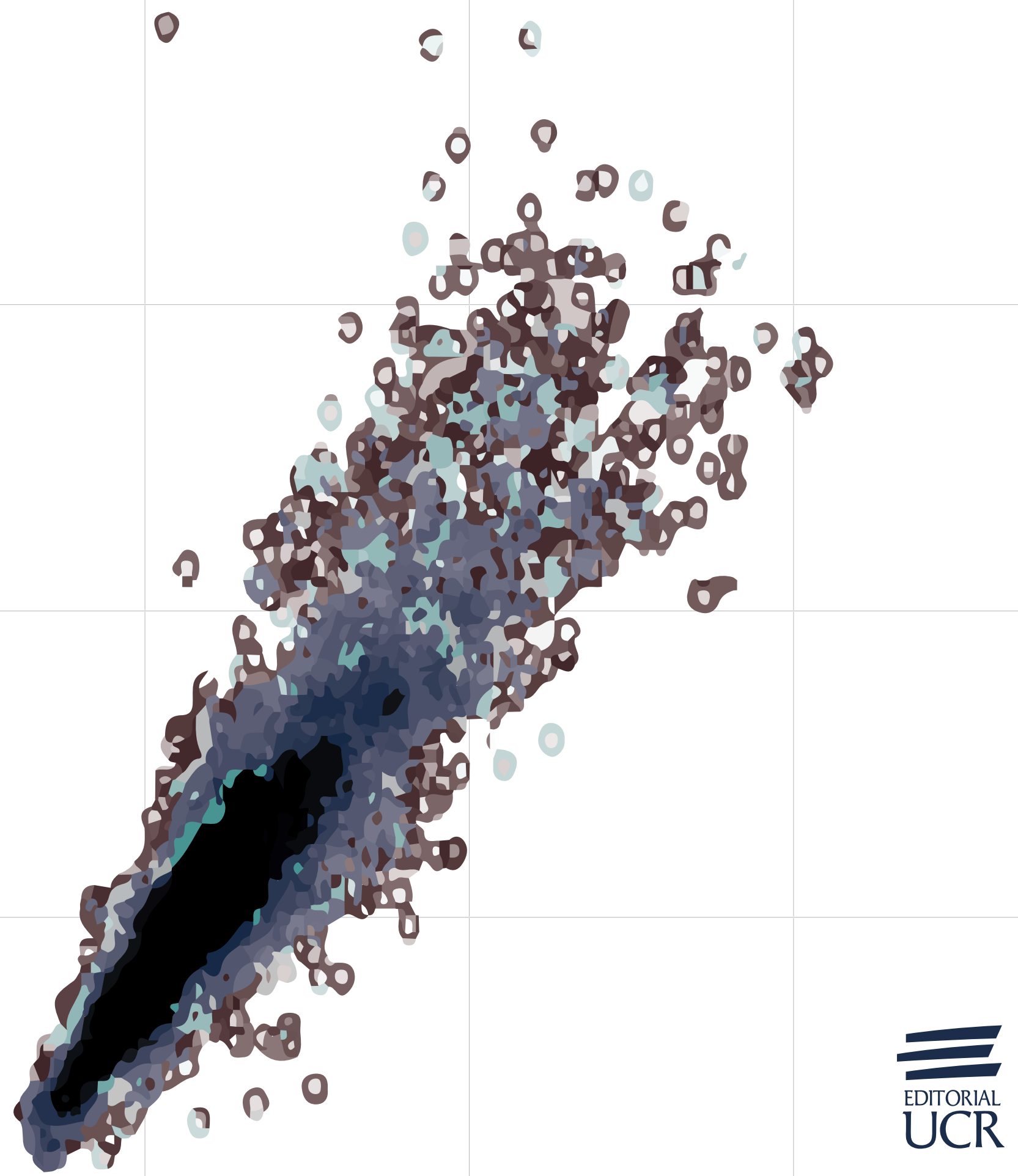




\title{
Método EBE de los elementos finitos para sistemas de gran tamaño y su aplicación en la Física Digital de Rocas
}

\author{
EBE finite element methdology for large scale systems applied to \\ digital rock Physics
}

\author{
Francisco José Benavides Murillo \\ Universidad Nacional de Costa Rica, Costa Rica. \\ Correos: fbenavides@id.uff.br
}

Recibido: 2 de febrero 2019

Aceptado: 5 de julio 2019

\begin{abstract}
Resumen
El método de los elementos finitos (FEM) es una técnica numérica ampliamente utilizada en física e ingeniería, que aproxima soluciones de ecuaciones diferenciales en derivadas parciales en dominios arbitrarios. En general, el paso final de esta técnica consiste en la resolución de un sistema lineal de la forma Ax=b, en el que la matriz A es dispersa y cuyo ancho de banda (número de filas no nulas en la matriz) depende del soporte de las funciones de forma definidas en los elementos. Cuando este número de elementos es muy grande, inclusive las representaciones en estructuras de datos eficientes para matrices dispersas son capaces de consumir en su totalidad la memoria de un computador poderoso. Por tanto, en este artículo se describe el método EBE (Element by Element), un truco computacional diseñado para implementar el método FEM sin utilizar la representación explícita de esta matriz. Se describe también un contexto en el que es necesaria esta implementación, en el área de la petrofísica digital de rocas, para estimar las propiedades elásticas de una muestra de roca a partir de imágenes tomográficas.
\end{abstract}

Palabras clave:

Análisis Numérico, Elemento Finito, Elemento por Elemento, Petrofísica, Propiedades elásticas

\begin{abstract}
The finite element method (FEM) is a numerical technique that estimates solutions of partial differential equations on arbitrary domains. It has been widely used to solve problems in Physics and Engineering. In general, the final step of this technique consists of a linear system of equations $A x=b$ in which the matrix A is sparse and its bandwidth depends on the finite element shape functions support. When the number of elements is large, even the efficient data structure sparse matrix representations can consume the entire computer memory. In this article, we describe a technique to solve these large-scale problems without explicitly representing this matrix. This computational trick is known as EBE (Element By Element). We also describe an application in which such kind of implementation is necessary, in the field of digital rock physics, to estimate the elastic coefficient of rock samples using micro-tomographic images.
\end{abstract}

Keywords:

Numerical Analysis, Finite Element, Element By Element, Petrophysics, Elastic properties

Esta obra está bajo una Licencia de Creative Commons. Atribución - No Comercial - Compartir Igual 


\section{INTRODUCCIÓN}

El método de los elementos finitos (FEM) es una técnica proyectiva, en la que una función $\varphi \in$ $X$ de un espacio de Hilbert $X$, es aproximada de manera óptima en un sub-espacio $U \subset X$ mediante su única proyección $\varphi_{U}$ (Kress, 1999). Por una parte, la función $\varphi$ es la solución de una ecuación diferencial en derivadas parciales sobre un dominio arbitrario, dominio que puede modelar un objeto físico real. Por otra parte $U$ es, en general, un sub-espacio de dimensión finita cuya base está formada por funciones de soporte compacto llamadas funciones de forma.

Tales funciones suelen ser definidas con base en subdominios o elementos geométricos (triángulos o hexaedros, por ejemplo). La aproximación minimiza la norma $\left\|\varphi-\varphi_{U}\right\|$ asociada a la forma variacional del problema, la cual se calcula en términos de productos internos en el espacio de funciones $U$ (Hughes, 2000). La forma variacional representa la energía asociada al problema físico modelado; por esta razón, el FEM es ampliamente utilizado en aplicaciones de ingeniería, en áreas tales como Mecánica de Materiales y Teoría de Elasticidad (Slaughter, 2002).

También, el FEM es utilizado en la simulación de procesos físicos en la Física de Rocas; ciencia que relaciona observaciones geofísicas obtenidas remotamente con las propiedades físicas subyacentes de las rocas, tales como composición, porosidad, permeabilidad, coeficientes elásticos, etc (Mavko et al., 2003).

Las propiedades mecánicas de las rocas son útiles para estimar sus procesos de deformación y son aplicadas en la Ingeniería de Petróleo para optimizar los métodos de perforación y extracción (Tiab and Donaldson, 2012). Estas propiedades pueden ser obtenidas a partir de ensayos de laboratorio, pero recientemente esto se ha hecho a partir de imágenes tomográficas de muestras de las rocas del reservatorio. Obtener tales imágenes para simular, en un computador, los procesos físicos del espacio poroso y mineral de la estructura rocosa es el paradigma de la Física Digital de las Rocas.

Cabe aclarar que este artículo se basa en el enfoque descrito en Garboczi y Day (1995) para estimar el módulo de Young o módulo de Elasticidad de una muestra de roca, digitalizada mediante una secuencia de imágenes tomográficas. Asimismo, utiliza la versión más simple de la técnica de los elementos finitos, utilizando apenas interpolaciones lineales entre los nodos y métodos clásicos de gradiente para resolver el sistema lineal asociado.

El interés principal de la investigación no está dirigido hacia la justificación del uso de los elementos finitos para resolver el problema, ni en proponer algún método novedoso para el FEM. Se considera que ese tipo de justificaciones ya han sido abordadas, por ejemplo en (Arns et al., 2002; Garboczi and Day, 1995; Makarynska et al., 2008; y Roberts y Garboczi, 2000). En dichos artículos, se utiliza FEM para estimar el módulo de Young, utilizando una cantidad muy grande de grados de libertad (definiendo un elemento por voxel), con sistemas de ecuaciones lineales que pueden llegar fácilmente a más de cien millones de incógnitas.

Asimismo, las investigaciones mencionadas no describen con detalle los aspectos computacionales

que hay que tener en cuenta a la hora de resolver sistemas de ese tamaño, lo que puede llevar a la 
conclusión errónea de que son necesarios super computadores de gran tamaño para su tratamiento. En realidad, sistemas de este orden pueden ser resueltos en Workstations comunes con la metodología que se describe en este artículo, la cual, de hecho, ha sido utilizada con éxito en (Leiderman et al., 2017). Cabe resaltar que el rango de aplicaciones se puede extender a otros tipos de problemas mecánicos (Betancur et al., 2018).

El truco computacional descrito en este trabajo no es novedoso, pero representa una marcada diferencia entre la utilización óptima de los recursos computacionales y la falsa necesidad de aumentar la capacidad del hardware con el fin de abordar este tipo de problemas.

Vale la pena remarcar que, debido a la existencia actual de una gran cantidad de implementaciones computacionales del FEM, tanto comerciales como libres, su utilización para estimar el módulo de Young a partir de imágenes parece una escogencia obvia, natural y preferible. Sin embargo, como se verá a lo largo de este artículo, la mayor parte de estos programas hacen implementaciones genéricas de los elementos finitos que no optimizan las características especiales del caso aquí presentado. De hecho, su utilización para simular un ensayo de laboratorio no es posible para tamaños relativamente grandes, que son normales en el contexto de la Física Digital de Rocas, y una implementación genérica del FEM agota fácilmente los recursos de la máquina.

De este modo, la implementación descrita en el presente artículo surge a raíz de la necesidad de simular numérica y computacionalmente un experimento de laboratorio (específicamente un ensayo de compresión uniaxial, como se describe en las siguientes secciones) con base en una secuencia de imágenes de una muestra de roca.

Tales imágenes representan, con precisión, la estructura del medio poroso, y permiten realizar múltiples ensayos virtuales no destructivos en la muestra. La utilización de imágenes para simular experimentos de laboratorio tiene algunas ventajas de rapidez, eficiencia y costo, pero también tiene el interés científico de conducir a una mejor comprensión del fenómeno físico modelado (Andrä et al., 2013). Entre los muchos ejemplos que se pueden citar, se puede considerar el trabajo de (Benavides et al., 2017), donde se propone una metodología computacional para mejorar la estimación de un parámetro de la roca, utilizando experimentos virtuales en imágenes tomográficas.

El resto del artículo se distribuye de la siguiente manera: en el marco teórico se hace una descripción sucinta del ensayo de compresión uniaxial; luego, se describe el método FEM desde el punto de vista de la implementación para este tipo de ensayo. Se aborda la resolución, por medio de FEM, de la ecuación elastostática, describiendo los operadores y la forma de calcularlos. Posteriormente, se describe el método EBE de manera detallada, tratando de definir, paso por paso, un algoritmo implementable. Se tratan de llenar los vacíos de otras descripciones menos detalladas, por ejemplo, (Leiderman et al., 2017; Ulrich et al., 1998. Luego se describen otros detalles computacionales, como la forma de paralelizar y validar; finalmente, se presentan las conclusiones. 


\section{MARCO TEÓRICO}

\subsection{Ecuación elastostática y el ensayo de compresión uniaxial}

La prueba de compresión uniaxial es un ensayo de laboratorio en el que se estima el coeficiente de Young de una muestra de roca (Hudson and Harrison, 2000). Este mide la compresibilidad homogénea equivalente a una muestra heterogénea de roca, compuesta por diferentes tipos de materiales con diferentes propiedades mecánicas y por un espacio de poros vacíos con geometría arbitraria. La prueba consiste en someter la muestra a una deformación fija u_y a lo largo del eje Y, manteniendo libertad de movimiento en los otros ejes (Figura 1, izquierda).

Para lograr tal deformación, se ha necesitado aplicar una presión en el área superior, dando como resultado una fuerza total $F$. Tal fuerza se relaciona con la deformación mediante la ecuación: $\frac{F}{A}=E \frac{u_{y}}{h}$, donde $E$ es el coeficiente de Young efectivo, $h$ es la altura de la muestra y $A$ es el área de la parte superior.

Dicha ecuación define la ley de Hooke en una dimensión. Para calcular esta fuerza se utiliza la solución del campo de desplazamiento de la ecuación elastostática. Con este campo, es posible calcular las presiones en la superficie superior de la muestra y, mediante integración numérica, calcular la fuerza en toda la superficie. Las condiciones de contorno son descritas mediante el desplazamiento $u_{y}$ y la fijación de la superficie inferior de la muestra. No hay otras presiones externas. Un modelo de muestra de roca (Figura 1, derecha) incluye gran cantidad de detalles del medio poroso, por lo que la malla debe ser fina. Habitualmente se utiliza un voxel por elemento, lo que produce sistemas de gran tamaño. Esto se aborda con más detalle en las siguientes secciones.
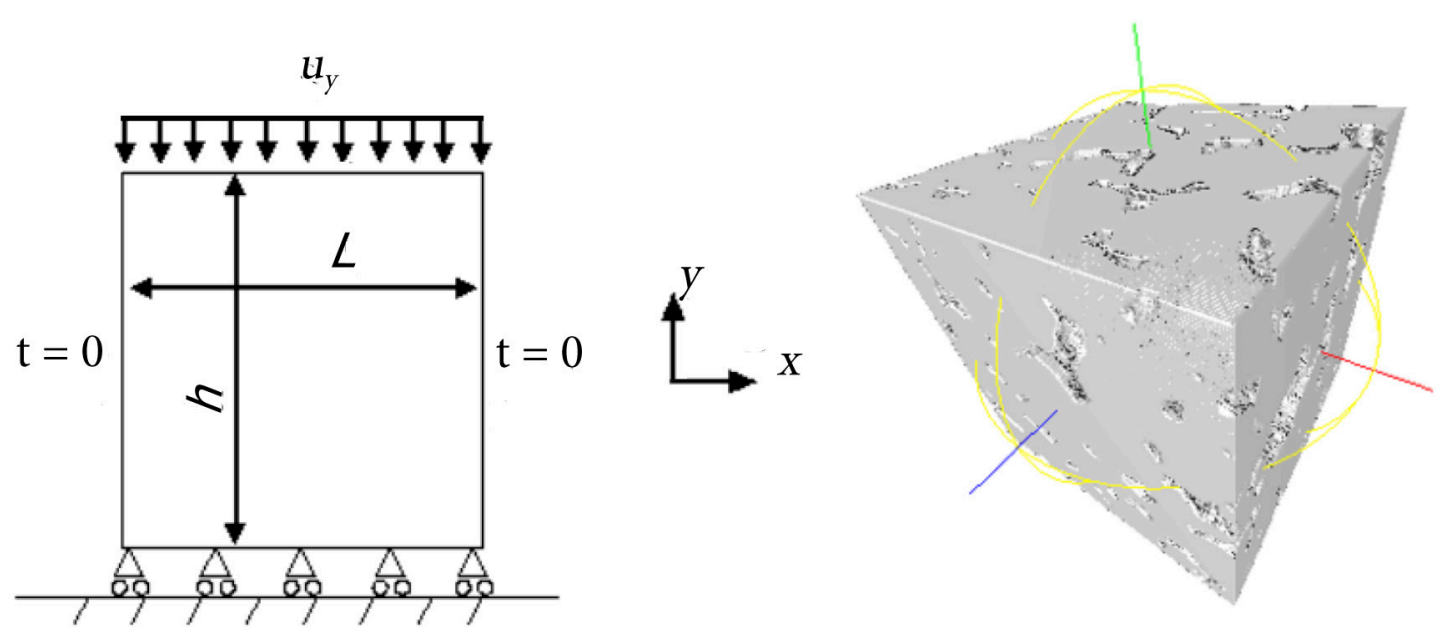

Figura 1. Izquierda: esquema de un ensayo de compresión uniaxial; derecha: Textura 3D de una muestra de roca, obtenido como una malla 3D. Nota: La resolución es de 1 micrómetro por voxel y la imagen es de 200x200x200 voxels 


\subsection{Resolución de la ecuación elastostática}

En esta sección se hace una breve presentación del método de los elementos finitos (FEM) en el contexto de la ecuación elastostática. El objetivo de esta sección es describir algunas ideas básicas, suficientes para explicar la técnica EBE. Se sigue de cerca la presentación de Hughes (2000), así como la de Garboczi and Day (1995). Lo aquí expuesto puede utilizarse para una implementación clásica del FEM.

Quizás la forma más directa de visualizar el FEM es como una técnica proyectiva, usada para resolver ecuaciones diferenciales y que culmina, en general, con un sistema lineal $K c=f$.

Se puede suponer $\boldsymbol{u}: \mathbb{R}^{3} \rightarrow \mathbb{R}^{3}$ es un campo vectorial con producto interno definido $a(\cdot, ;)$. En el método de los elementos finitos, cada una de las entradas de $\mathrm{u}$, denotada por $u i, i=1,2,3$ es aproximada mediante una combinación lineal de funciones de forma $\left\{N_{A}: \mathbb{R}^{3} \rightarrow \mathbb{R}, A=1, \ldots \ldots, N\right\}$ y una función $g_{i}$ asociada a las condiciones de contorno del dominio. Si $\tilde{u}$ es tal aproximación, entonces cada una de sus entradas $\tilde{u}_{i}$ está dada por:

$$
\tilde{u}_{i}=\sum_{A=1}^{N} c_{A i} N_{A}+g_{i}
$$

Esto produce $3 N$ incógnitas $c_{A i}$. Si $e_{1}, e_{2}, e_{3}$ son los vectores de la base Euclídea en $\mathbb{R}^{3}$, entonces, al aplicar la propiedad ortográfica a esta proyección, se obtiene:

$$
a\left(N_{A} e_{j}, u-\tilde{u}\right)=0 \quad j=1,2,3
$$

La forma de elegir el operador $a(\cdot, ;)$ está asociada con la ecuación diferencial por resolver. En este caso, se trata de la ecuación elastostática, que en notación inicial tiene la forma:

$$
\sigma_{i j, j}=0
$$

Aquí, el doble índice denota sumatoria y la coma, diferenciación con respecto a la variable indizada. Los valores de $\sigma_{i j}$ conforman el tensor de tensiones, simétrico, de tamaño $3 \times 3$. Este tipo de notación es empleada por Hughes (2000), y se caracteriza por ser compacta y breve.

Ahora bien, hallar la solución consiste en obtener el campo de dislocamiento $u=\left(u_{1}, u_{2}, u_{3}\right)$ en todo el dominio. Este campo está relacionado con el tensor de tensiones por medio de la ley de Hooke (Slaughter, 2002):

$$
\sigma_{i j}=\mathrm{c}_{i j k l} \in_{k l}
$$

Donde las constantes $\mathrm{c}_{i j k l}$ corresponden al tensor de rigidez, dependiente del tipo de material y $\in_{k l}$ es el tensor simétrico de desplazamientos, definido en términos de los desplazamientos $u_{l}$ como: 


$$
\epsilon_{k l}=u_{(k, l)}=\frac{1}{2}\left(u_{k, l}+u_{l, k}\right)
$$

En materiales isotrópicos el tensor $\mathrm{c}_{i j k l}$ es simplificado en una matriz $6 \times 6$ sacando provecho de la simetría de los tensores de desplazamiento y el de tensiones. La forma de la Ley de Hooke para este caso es:

$$
\left(\begin{array}{l}
\sigma_{11} \\
\sigma_{22} \\
\sigma_{33} \\
\sigma_{23} \\
\sigma_{13} \\
\sigma_{12}
\end{array}\right)=\left(\begin{array}{cccccc}
2 \mu+\lambda & \lambda & \lambda & 0 & 0 & 0 \\
\lambda & 2 \mu+\lambda & \lambda & 0 & 0 & 0 \\
\lambda & \lambda & 2 \mu+\lambda & 0 & 0 & 0 \\
0 & 0 & 0 & \mu & 0 & 0 \\
0 & 0 & 0 & 0 & \mu & 0 \\
0 & 0 & 0 & 0 & 0 & \mu
\end{array}\right)\left(\begin{array}{l}
\epsilon_{11} \\
\epsilon_{22} \\
\epsilon_{33} \\
\epsilon_{23} \\
\epsilon_{13} \\
\epsilon_{12}
\end{array}\right)
$$

Donde $\mu$ y $\lambda$ son las constantes de Lamé del material modelado. Para que el problema esté bien planteado, se deben definir las condiciones de contorno que se clasifican en dos tipos: condiciones de Neuman y condiciones de Drichlet (Hughes, 2000). En mecánica, estas condiciones pueden ser interpretadas de la siguiente forma:

1. Condiciones de contorno en las que el desplazamiento es conocido $T_{G}$

2. Condiciones de contorno en las que es conocida la tensión externa aplicada $T_{H}$

En este caso, no existen tensiones externas, pero sí un desplazamiento conocido, tal y como se corresponde en la prueba de compresión uniaxial. De esta manera, las condiciones de contorno son: $u_{i}=q_{i}$ para los desplazamientos conocidos y $\sigma_{i j} n_{j}=0$ en los puntos de frontera donde no se aplican presiones externas. El vector $\boldsymbol{n}=\left(n_{1}, n_{2}, n_{3}\right)$ es un vector normal superficial. En la expansión de la ecuación (1), la función $g_{i}$ estará asociada a las condiciones de contorno $T_{G}$.

Para la implementación computacional del operador $a(\cdot ;)$, se define la matriz de derivadas para la función de forma indizada por $A$ como $D_{A}$ :

$$
D_{A}=\left(\begin{array}{ccc}
N_{A, 1} & 0 & 0 \\
0 & N_{A, 2} & 0 \\
0 & 0 & N_{A, 3} \\
0 & N_{A, 3} & N_{A, 2} \\
N_{A, 3} & 0 & N_{A, 1} \\
N_{A, 2} & N_{A, 1} & 0
\end{array}\right)
$$

La matriz de integrales para los índices $A$ y $B, K_{A B}=K_{A B}=\int D_{A}^{t} K_{e} D_{B}$ se calcula en todo el dominio de integración. La matriz $\mathrm{K}_{e}$ está definida por la ecuación (4). El producto interno $a\left(N_{A} e_{i}, N_{B} e_{j}\right)$, 
que depende de los índices $A$ y $B$ de las funciones de forma y de los índices $i$ y $j$ contenidos en el conjunto $\{1,2,3\}$, se define como la entrada $(i, j)$ de la matriz $K_{A B}$. Esto puede escribirse como $a\left(N_{A} \boldsymbol{e}_{\boldsymbol{i}}, N_{B} \boldsymbol{e}_{\boldsymbol{j}}\right)=\boldsymbol{e}_{\boldsymbol{i}}^{\boldsymbol{t}} K_{A B} \boldsymbol{e}_{\boldsymbol{j}}$. Con este montaje, lo que ocurre es que para cualesquier campo vectorial $\boldsymbol{W}=W_{1} e_{1}+W_{2} e_{2}+W_{3} e_{3} y \boldsymbol{V}=V_{1} e_{1}+V_{-} 2 e_{2}+V_{3} e_{3}$ se tendrá $a(\boldsymbol{W}, \boldsymbol{V})=\int W_{(i, j)} c_{i j k l} V_{(k, l)}$. Volviendo a la propiedad ortográfica de la ecuación (2), se tiene que:

$$
a\left(N_{A} e_{j}, u-\tilde{u}\right)=a\left(N_{A} e_{j}, u\right)-a\left(N_{A} e_{j}, \tilde{u}\right)=0
$$

Donde $a\left(N_{A} e_{j}, u\right)=\int\left(N_{A} e_{j}\right)_{(p, q)} c_{p q k l} u_{(k, l)}=\int\left(N_{A} e_{j}\right)_{(p, q)} \sigma_{p q}$

Por el Teorema de Green, se obtiene la siguiente separación (Hughes, 2000):

$$
\int\left(N_{A} e_{j}\right)_{(p, q)} \sigma_{p q}=-\int N_{A} \sigma_{j q, q}+\oint_{T} N_{A} \sigma_{j q} n_{q}
$$

La integral de contorno, por su parte, es evaluada en $\mathrm{T}=\mathrm{T}_{\mathrm{H}} \mathrm{UT}_{\mathrm{G}}$. Suponiendo que las funciones de forma $N_{A}$ son iguales a cero en $T_{G}$ y que no existen tensiones externas, el resultado de esta suma es igual a 0, debido a la ecuación (3). La ecuación (2) se simplifica de la siguiente manera:

$$
a\left(N_{A} \boldsymbol{e}_{\boldsymbol{j}}, \widetilde{\boldsymbol{u}}\right)=a\left(N_{A} \boldsymbol{e}_{\boldsymbol{j}}, \sum_{i=1}^{3} \mathfrak{u}_{i} \boldsymbol{e}_{\boldsymbol{i}}\right)=0
$$

Utilizando ahora la expansión de la ecuación (1) en este resultado, se obtiene:

$$
\sum_{i=1}^{3} \sum_{B=1}^{N} c_{A i} a\left(N_{A} \boldsymbol{e}_{\boldsymbol{j}}, N_{B} \boldsymbol{e}_{\boldsymbol{i}}\right)=-a\left(N_{A} \boldsymbol{e}_{\boldsymbol{j}}, \boldsymbol{g}\right)
$$

En el FEM, se asume que g es combinación lineal de funciones de soporte compacto (similares a las de forma) que no se anulan en el contorno $T_{G}$. La resolución de este sistema lineal $K_{c}=f$, donde $K$ es la matriz de productos internos $a\left(N_{A} e_{j}, N_{B} e_{i}\right)$ y $f$ tiene por entradas los productos $-a\left(N_{A} e_{j}, g\right)$, es el paso final del método. K es de tamaño $3 N \times 3 N$. Las funciones de forma son cero en la mayoría de los elementos del dominio, de modo que para cada $A$, existe solo un número limitado de índices $B$ para los cuales el producto $a\left(N_{A} e_{j}, N_{B} e_{i}\right)$ no es cero. El número máximo de índices para el que esto ocurre es el ancho de banda de la matriz de rigidez $K$. El sistema de ecuaciones es resuelto mediante la técnica iterativa del gradiente conjugado (Trefethen and Bau, 1997). 


\section{DESCRIPCIÓN DEL ALGORITMO EBE}

\subsection{Implementación del método EBE}

Para evitar los costos computacionales de representar la matriz de productos internos $K_{i j}=a\left(N_{A} e_{j}, N_{B} \boldsymbol{e}_{i}\right)$, se utiliza el método EBE. Esta idea ya fue utilizada en la estimación de los coeficientes elásticos de huesos trabeculares (van Rietbergen et al., 1995). En su investigación, los autores mencionan que la matriz de productos internos $K$ no precisa ser montada, y que se puede aprovechar la regularidad del modelo (todos los elementos tienen el mismo tamaño) para ahorrar memoria y procesamiento. Asumen que las propiedades de la fase sólida de todos los elementos son idénticos, lo que, como se verá a continuación, no es necesario. Los autores tampoco aplican ninguna precondición a esta matriz. En el algoritmo que se describe a continuación también se aplica, de manera opcional, un precondicionador de Jacobi (Trefethen and Bau, 1997).

La estrategia EBE está ligada con el proceso iterativo que obtiene la solución del sistema lineal $K_{C}=f$. El método de los gradientes conjugados (así como cualquier otra técnica iterativa aplicada a este caso), se basa en sucesivos productos de matriz por vector $s=K r$ que van mejorando la aproximación de la solución en cada iteración. Aunque en esta técnica numérica se efectúan otros cálculos vectoriales, este producto matricial es el proceso más pesado computacionalmente. En lugar de calcular esta matriz de una sola vez, la estrategia EBE toma como entrada el vector $\boldsymbol{r}$ y lo va multiplicando por los productos internos $a\left(N_{A} e_{j}, N_{A} e_{i}\right)$ elemento por elemento, actualizando al mismo tiempo, el vector resultante $\boldsymbol{s}$.

En otras palabras, la matriz $K$ es ensamblada, por partes, en cada iteración, actuando inmediatamente sobre el vector resultante, sin almacenar nunca en memoria todos los valores de la estructura matricial.

La tarea de calcular los productos internos de las funciones de forma en cada iteración del método del gradiente conjugado es más optimizable cuando la malla es regular o parcialmente regular. Es decir, cuando está conformada por un conjunto limitado de clases de elementos. El almacenamiento en memoria de los productos internos asociados a cada elemento requiere únicamente una matriz $24 \times 24$.

Dicho producto depende de las constantes de Lamé y la geometría del elemento. En física de rocas, las muestras en general se consideran bifásicas (solo tienen fase sólida y poro), pues el mayor interés se centra en el estudio del espacio de poro. Los elementos son cubos del mismo tamaño, uno por cada voxel. Por esa razón, la matriz de productos internos sólo debe ser calculada una vez. Finalmente, para mejorar la velocidad de convergencia, se utiliza el precondicionador de Jacobi, de modo que los elementos de la diagonal de K son unitarios. Resumiendo, el método EBE requiere del cálculo previo de la matriz de productos internos $P_{k}=a\left(N_{A} \boldsymbol{e}_{j}, N_{A} \boldsymbol{e}_{i}\right)_{i j}$ para cada tipo de elemento. Cada una de estas matrices tiene tamaño $24 \times 24$. El producto vectorial $\mathrm{Kr}$ donde $K$ es la matriz de rigidez del sistema, se calcula iterando en cada elemento $E_{w}$ con base en el siguiente algoritmo, que aprovecha también la simetría de la matriz de rigidez $K$ : 
Entrada: vector $\boldsymbol{r}$, matrices de productos internos $P_{w}$ (para cada elemento $E_{w}$ )

Salida: vector $\boldsymbol{s}=K \boldsymbol{r}$. Vector precondicionador $\boldsymbol{d}$.

$$
\text { Inicializar } s=0 \text {. }
$$

Iterar sobre todos los elementos $E_{w}$

Para $A=1,2, \ldots, 8$, índices de los nodos de $E_{w}$

Para $B=A, \ldots, 8$, índices de los nodos de $E_{w}$

Para $i_{1}=1,2,3$ e $i_{2}=1,2,3$

Sea $p$ el índice de la fila de $K$ correspondiente al nodo $A$ y al grado de libertad $i_{1}$.

Sea $q$ el índice de la fila de $K$ correspondiente al nodo $B$ y al grado de libertad $i_{2}$.

Sea $v=P_{w}\left[3(A-1)+i_{1}, 3(B-1)+i_{2}\right]$

$s[p]=s[p]+\boldsymbol{r}[q] \cdot v$

Si $A \neq B$ entonces $s[q]=s[q]+\boldsymbol{r}[p] \cdot v$

Si $\mathrm{p}=\mathrm{q}$ se actualiza el precondicionador $d[p]=d[p]+v$ (esto únicamente se realiza durante la primera iteración, guardando en memoria el precondicionador $\boldsymbol{d}$ ).

Multiplicar cada entrada $s[i]$ por $1 / \boldsymbol{d}[i]$ (precondicionador de Jacobi).

\subsection{Paralelización}

Con el objeto de acelerar las operaciones computacionales, el trabajo que se realiza en cada elemento en el algoritmo descrito en la sección anterior debe ser ejecutado en paralelo. No es posible distribuir los elementos entre cada procesador de manera arbitraria, ya que dos elementos distintos, siendo procesados en procesadores diferentes, pueden intentar actualizar la misma entrada del vector resultante $\boldsymbol{s}$.

Para evitar lo anterior, es necesario separar los elementos en grupos diferentes, de modo que no exista intersección de los nodos de los elementos de cada grupo. Los grupos se procesan secuencialmente pero cada grupo se procesa en paralelo, pues no existen colisiones. En una malla regular tridimensional en la que cada voxel es un elemento, son necesarios únicamente 8 grupos 
diferentes. Esta división se lleva a cabo numerando cada voxel de la imagen de manera secuencial. Para el elemento asociado al voxel $j$, el grupo al que pertenece está dado simplemente por $j$ ( $\bmod$ 8). Este tipo de paralelización puede ser llevada a cabo tanto en la CPU como en un procesador gráfico (GPU) con suficiente memoria.

En la Figura 2 se muestra el esquema de división de grupos y numeración para la paralelización. En el caso ilustrado se actualizan los productos internos correspondientes a los elementos numerados con los valores de $j$ tales que $j(\bmod 8)=1$, coloreados en rojo. Los productos internos de las funciones de forma dentro de esos elementos no actualizan nunca la misma fila del vector resultante, porque los nodos nunca coinciden en cada elemento. Por esta razón, todos esos elementos en rojo pueden ser procesados en paralelo, sin necesidad de utilizar comandos de exclusión o MUTEXes (Reif and Rajasekaran, 2008).

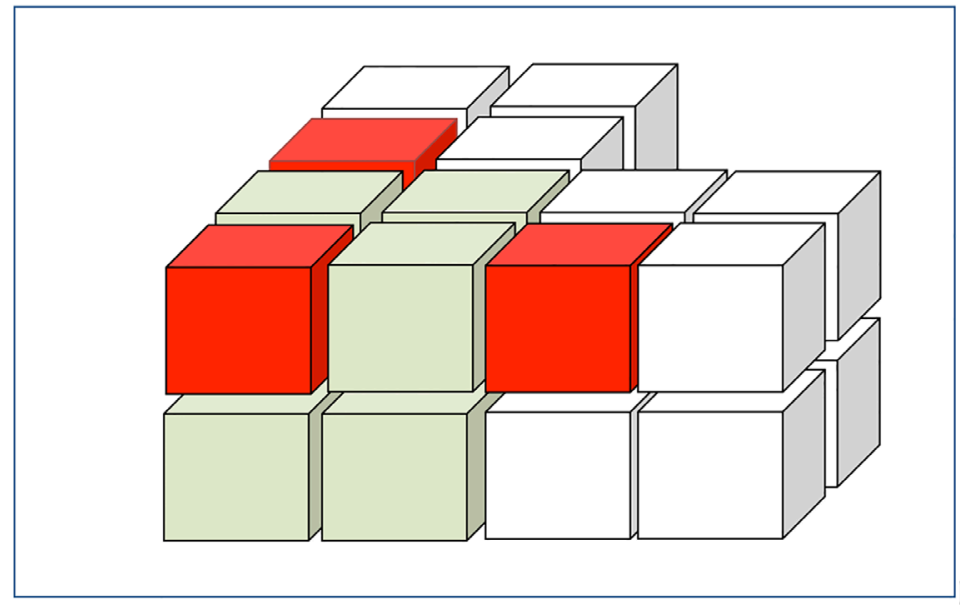

Figura 2. Distribución paralela de los elementos durante una iteración

Se muestran tres grupos de 8 voxels donde se realizan los productos internos de las funciones de forma que no se nulifican en los voxels coloreados en rojo. Es de notar que estos voxels coloreados no tienen nodos en común, por tanto no actualizan nunca la misma fila del vector resultante. Los otros 7 grupos son trabajados en paralelo en pasos subsecuentes.

Es importante recalcar que la metodología propuesta aquí simplemente posibilita la resolución de la ecuación elastostática en casos donde el número de elementos es tan grande que no puede ser administrado por un software FEM comercial. Pero el resultado final entre montar la matriz K y no hacerlo debería ser exactamente el mismo.

Por esa razón, una forma de validar esta metodología consiste en comparar el campo de dislocamiento obtenido, con el de un software comercial (en una malla pequeña, Figura 3). Una prueba de este tipo sería suficiente para validar la implementación, pero también es posible utilizar una malla grande (por ejemplo, 400×400×400) sin poros, comparando el módulo de Young obtenido con el que fue utilizando en cada elemento. El resultado debería ser el mismo. 


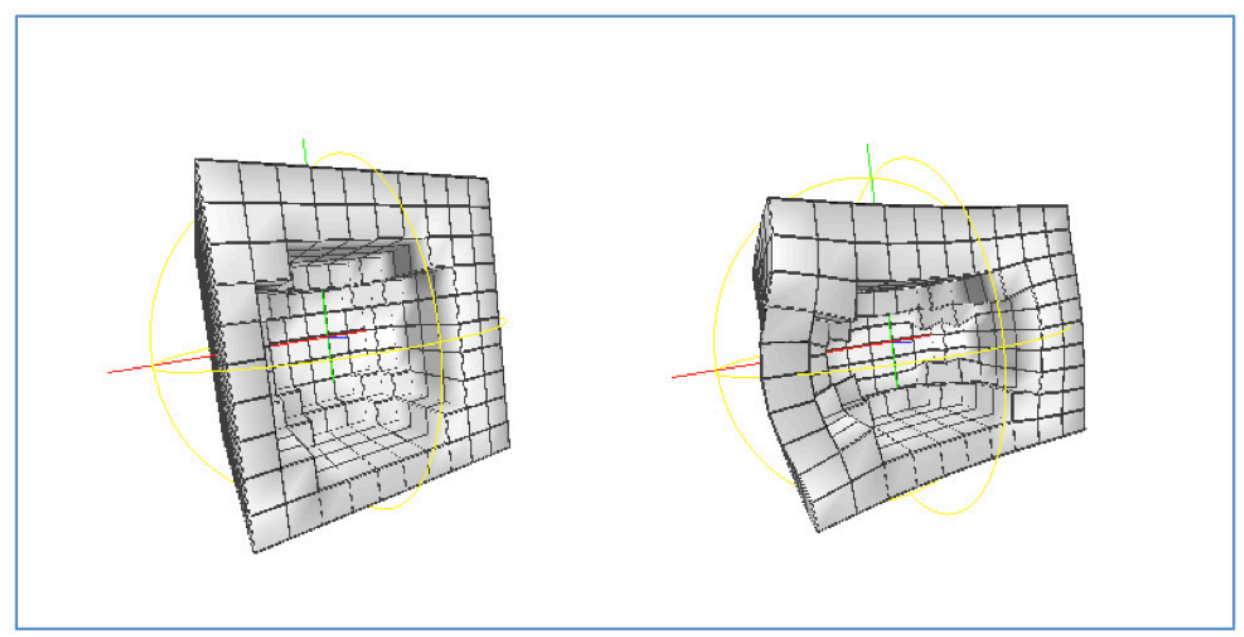

Figura 3. Izquierda: malla pequeña, porosa y regular; derecha: ensayo de compresión uniaxial aplicado a la malla, usando FEM

\section{VALIDACIÓN}

La eficiencia y velocidad del truco computacional expuesto en el presente trabajo depende de detalles de implementación tales como el hardware utilizado, el lenguaje de programación en el que se desarrolla el método, la forma en que se organiza la disposición de los elementos y los nodos en la memoria, las particularidades del medio poroso representado.

Se ha pretendido que el algoritmo descrito en la sección 3-1 no dependa de estos factores. Asimismo, se considera relevante observar que la implementación, realizada en C++ y ejecutada en una Workstation Dell XPS (con 16 GB de RAM y 8 procesadores en hyperhtread), procesa imágenes de tamaño 400×400×400 con más de 190 millones de grados de libertad en poco más de 25 minutos.

Dicho experimento sería imposible de resolver utilizando el método FEM representando explícitamente la matriz de rigidez sin hacer uso de una memoria RAM de más de 120 GB. Por el contrario, con el método EBE es necesario apenas un 5\% de esta memoria. No obstante, se considera que es posible aún mejorar múltiples detalles de implementación y obtener mejores rendimientos.

Las consideraciones antes expuestas, de tipo cualitativo, no deberían ser una guía para la validación o la confirmación de una implementación específica, sino apenas una orientación para quien necesite crear un software de elemento finito con características similares a las expuestas en el presente trabajo.

Además, es importante notar que lo que se ha descrito aquí es apenas es una técnica de implementación, cuyos resultados deberían ser totalmente equivalentes a los obtenidos con un método FEM tradicional, con software comercial o libre, bajo las mismas condiciones. Por ejemplo, el número de iteraciones del gradiente conjugado usando EBE, es el mismo que se obtiene con la representación explícita de la matriz, pues la novedad de este algoritmo es que se está alterando es la forma de representar la matriz de forma y la técnica para calcular el producto matriz-vector. En general, el método EBE es más lento que el explícito, pero solo pueden realizarse 
tales comparaciones en imágenes de tamaño máximo de 100×100×100 en el computador descrito. La diferencia en velocidad es aproximadamente de 1 a 5.

En el contexto de la estimación del módulo de Young para medios porosos, es posible validar la implementación utilizando el trabajo de (Hashin, 1983). Suponiendo una muestra bifásica con constantes de compresibilidad y elasticidad transversal $K_{S}, \mu_{S}$ para la matriz y $K_{P}$, $\mu_{P}$ para las inclusiones porosas, entonces los módulos de compresibilidad y elasticidad transversal $K$, $\mu$ de la muestra están dados por:

$$
\begin{aligned}
& K=K_{S}+p P^{S P}\left(K_{P}-K_{S}\right) \\
& \mu=\mu_{S}+p Q^{S P}\left(\mu_{P}-\mu_{S}\right)
\end{aligned}
$$

Donde

$$
P^{S P}=\frac{3 K_{S}+4 \mu_{S}}{3 K_{P}+4 \mu_{S}}, Q^{S P}=\frac{\mu_{S}+F_{S}}{\mu_{P}+F_{S}} \mathrm{y} F_{S}=\frac{\mu_{S}}{6} \cdot \frac{9 K_{S}+8 \mu_{S}}{K_{S}+2 \mu_{S}}
$$

y $p$ es la porosidad o razón volumétrica de las inclusiones. El módulo de Young equivalente se obtiene utilizando estas constantes (Slaughter, 2002):

$$
E=\frac{9 K \mu}{3 K+\mu}
$$

Estas ecuaciones son válidas bajo la suposición de que las inclusiones porosas dentro de la muestra son esféricas y están diluidas dentro de la matriz. Esto significa que la distribución de los poros es uniforme (isotropía) y que la tensión interna en un poro no está influenciada por los otros poros. Este es el comportamiento de una matriz porosa infinita (Roberts and Garboczi, 2002).

Estas fuertes condiciones pueden ser reproducidas en el espacio discreto de las imágenes, usando esferas discretas de Bressenham (Bresenham, 1965), distribuidas simétricamente en la matriz (como se ilustra en la Figura 4, izquierda). Los resultados del ensayo de compresión en esta estructura se ajustan muy bien a las ecuaciones teóricas (6) y (7) (Figura 4, derecha).

Para esta validación se ha utilizado $\lambda_{P}=\frac{\lambda_{S}}{3}=1$ y $\mu_{P}=\frac{\mu_{S}}{2}=\frac{1}{4}$ en unidades normalizadas para las fases sólida y porosa. El error relativo se incrementa con la porosidad, hasta que la hipótesis de poros diluidos de Hashin deja de cumplirse (porosidad mayor a 0.6). Los errores relativos de los otros casos, para porosidades menores a 0.6 , aunque muy pequeños (menores al $1 \%$ ), no son nulos. Esto se debe a los errores de discretización del modelado de las inclusiones esféricas en un espacio basado en voxels.

Dicho esquema de validación es útil cuando es imposible realizar un ensayo de compresión basado en una implementación comercial tradicional, debido al tamaño y número de las imágenes. Los resultados mostrados se han ejecutado en 200 imágenes de tamaño $200 \times 200$, lo que produce 8 millones de elementos. 


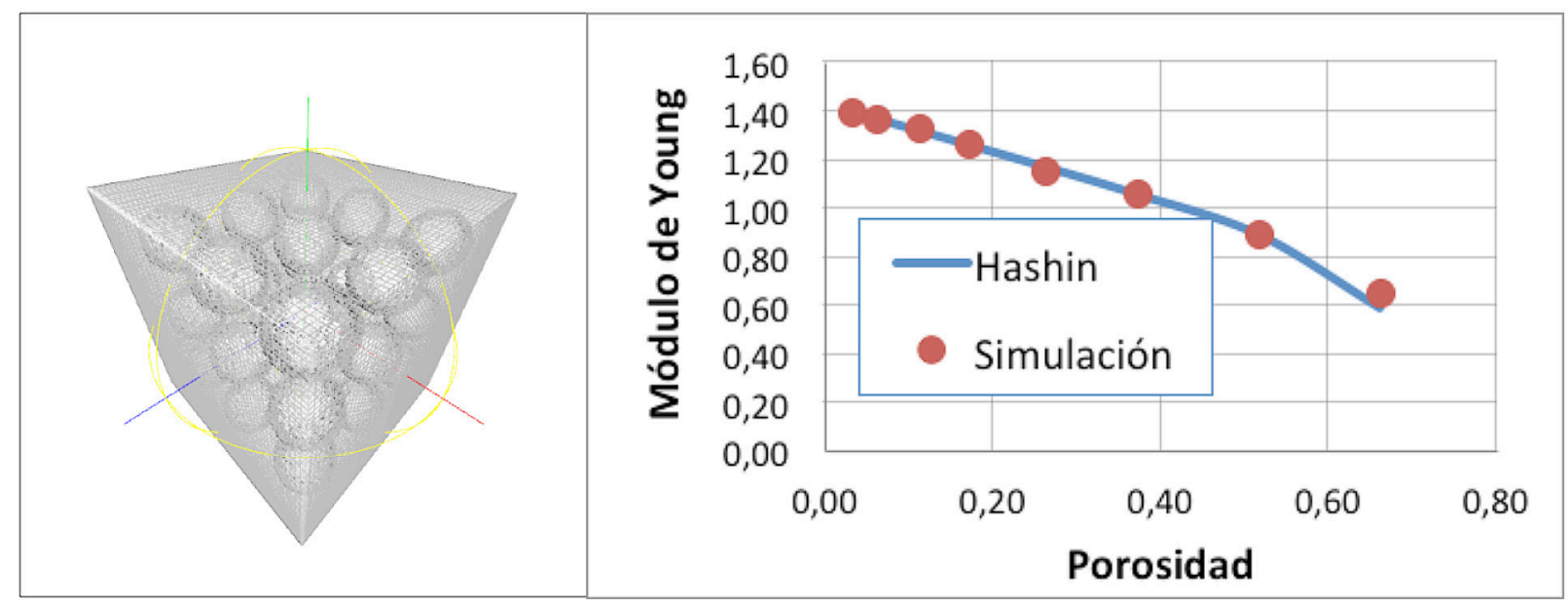

Figura 4. Izquierda: distribución uniforme de un arreglo de 27 poros esféricos dentro de una matriz sólida; derecha: esferas.

Nota: Módulo de Young normalizado obtenido a partir de un ensayo de compresión aplicado a la muestra, alterando el radio de las esferas para obtenerlo como una función de la porosidad. Línea continua, módulo de Young teórico, obtenido como función de la porosidad utilizando las ecuaciones (6) y (7).

\section{CONCLUSIONES}

Como se ha dicho anteriormente, este tipo de implementación de elementos finitos es útil en contextos similares a lo que se ha descrito aquí: Física Digital de Rocas para la estimación de parámetros de modelos muy heterogéneos, utilizando FEM con muchos elementos iguales y regulares.

Además, se adapta fácilmente para condiciones de contorno periódicas, en donde se supone que la muestra está sumergida en un medio infinito y regular. Su aplicación en computadores de mayor poder permite resolver imágenes de orden $1000^{3}$ o inclusive $2000^{3}$ (en los que el número de grados de libertad está en el orden de billones).

Sin duda, el método EBE es la única opción viable para implementar la técnica clásica de los elementos finitos cuando el número de elementos y de grados de libertad producen una matriz de rigidez que sobrepasa las capacidades físicas de un computador.

La gran cantidad de implementaciones comerciales existentes para resolver problemas numéricos mediante el FEM puede causar la impresión de que no es necesario programar otro tipo de implementaciones y apenas utilizar las que ya existen, pues de todos modos ya están optimizadas y depuradas.

En este artículo se ha descrito un contexto en el que una implementación genérica comercial no es aplicable. Bajo estas circunstancias, un usuario de estas aplicaciones puede verse en una situación en la que los requerimientos de hardware son imposibles o tienen un costo muy alto, por lo que el esfuerzo de "reinventar la rueda" y crear una implementación propia tiene una ventaja importante desde el punto de vista del costo-beneficio. 


\section{REFERENCIA BIBLIOGRÁFICAS}

Andrä, H., et al. (2013). Digital rock physics benchmarks-Part I: Imaging and segmentation. Comput. Geosci. 50, 25-32. https://doi.org/10.1016/j.cageo.2012.09.005

Arns, C.H., Knackstedt, M.A., Pinczewski, W.V. y Garboczi, E.J. (2002). Computation of linear elastic properties from microtomographic images: Methodology and agreement between theory and experiment. Geophysics 67, 1396-1405. https://doi.org/10.1190/1.1512785

Benavides, F., Leiderman, R., Souza, A., Carneiro, G. y Bagueira, R. (2017). Estimating the surface relaxivity as a function of pore size from NMR T2 distributions and micro-tomographic images. Comput. Geosci. 106. https://doi.org/10.1016/j.cageo.2017.06.016

Betancur, A., Anflor, C., Pereira, A. y Leiderman, R. (2018). Determination of the Effective Elastic Modulus for Nodular Cast Iron Using the Boundary Element Method. Metals (Basel). 8, 641. https://doi. org/10.3390/met8080641

Bresenham, J.E. (1965). Algorithm for computer control of a digital plotter. IBM Syst. J. 4, 25-30. https:// doi.org/10.1147/sj.41.0025

Garboczi, E.J. y Day, A.R. (1995). An algorithm for computing the effective linear elastic properties of heterogeneous materials: Three-dimensional results for composites with equal phase poisson ratios. J. Mech. Phys. Solids 43, 1349-1362. https://doi.org/10.1016/0022-5096(95)00050-S

Hashin, Z. (1983). Analysis of Composite Materials---A Survey. J. Appl. Mech. Asme 50. https://doi. org/10.1115/1.3167081

Hudson, J.A. y Harrison, J.P. (2000). Engineering Rock Mechanics: An Introduction to the Principles. Elsevier Science, 86-96.

Hughes, T.J.R. (2000). The Finite Element Method: Linear Static and Dynamic Finite Element Analysis. Dover Civil and Mechanical Engineering, 57-107.

Kress, R. (1999). Linear Integral Equations, in: Applied Mathematical Sciences. Springer New York, 218-245.

Leiderman, R., Pereira, A.M.B., Benavides, F.M.J., Silveira, C.S., Almeida, R.M.R. y Bagueira, R.A. (2017). PERSONAL COMPUTER-BASED DIGITAL PETROPHYSICS. Rev. Bras. Geofísica 35, 95-107. https://doi.org/10.22564/rbgf.v35i2.891

Makarynska, D., Gurevich, B., Ciz, R., Arns, C.H. y Knackstedt, M.A. (2008). Finite element modelling of the effective elastic properties of partially saturated rocks. Comput. Geosci. 34, 647-657. https://doi. org/http://dx.doi.org/10.1016/j.cageo.2007.06.009

Mavko, G., Mukerji, T. y Dvorkin, J. (2003). The Rock Physics Handbook: Tools for Seismic Analysis of Porous Media, in: Stanford-Cambridge Program. Cambridge University Press, 21-81.

Reif, J. y Rajasekaran, S. (2008). Handbook of Parallel Computing: Models, Algorithms, and Applications, First Edit. ed. Chapman \& Hall/CRC Computer and information science series. 
Roberts, A.P. y Garboczi, E. (2000). Elastic Properties of Model Porous Ceramics. J. Am. Ceram. Soc.

Roberts, A.P. y Garboczi, E.J. (2002). Computation of the linear elastic properties of random porous materials with a wide variety of microstructure. Proc. R. Soc. A Math. Phys. Eng. Sci. 458, 1033-1054. https:// doi.org/10.1098/rspa.2001.0900

Slaughter, W.S. (2002). The Linearized Theory of Elasticity. Birkhäuser Boston, 387-426.

Tiab, D. y Donaldson, E.C. (2012). Petrophysics: Theory and Practice of Measuring Reservoir Rock and Fluid Transport Properties. EngineeringPro Collection. Elsevier Science, 554-660.

Trefethen, L.N. y Bau, D. (1997). Numerical Linear Algebra. Other Titles in Applied Mathematics. Society for Industrial and Applied Mathematics (SIAM, 3600 Market Street, Floor 6, Philadelphia, PA 19104), 293-303.

Ulrich, D., Van Rietbergen, B. y Weinans, H., R P. (1998). Finite element analysis of trabecular bone structure: A comparison of image-based meshing techniques. J. Biomech. 31, 1187-1192. https://doi.org/10.1016/ S0021-9290(98)00118-3

Van Rietbergen, B., Weinans, H., Huiskes, R. y Odgaard, A. (1995). A new method to determine trabecular bone elastic properties and loading using micromechanical finite-element models. J. Biomech. 28, 69-81. https://doi.org/http://dx.doi.org/10.1016/0021-9290(95)80008-5 